\title{
HOMOTHETIC MAPPINGS OF RIEMANN SPACES
}

\author{
M. S. KNEBELMAN
}

1. Introduction. A homothetic mapping dilates any figure into a similar figure; it is therefore conformal. T. Sumitomo [1] proved that in a positive definite, compact orientable Riemann space such a mapping either does not exist or reduces to a motion; that is, the dilation constant is zero. The main purpose of this note is to prove Theorem 2.1 which gives some group theoretic insight into the reason for the scarcity of homothetic mappings. The technique used is that of Lie differentiation $[2 ; 3]$.

2. Homothetic mappings. Let $V_{n}$ be a Riemann space with fundamental metric $g_{i j}(x)$. Let $\xi^{i}(x)$ be a vector field defining a one parameter Lie group and $L$ be the symbol of Lie differentiation based on $\xi^{i}(x)$. The condition that $\xi^{i}$ define a homothetic mapping of $V_{n}$ is

$$
L g_{i j} \equiv \xi_{i, j}+\xi_{j, i}=c g_{i j}
$$

where $\xi_{i, j}$ is the covariant derivative of $\xi_{i}$ and $c$ is a constant $\neq 0$. (If $c=0$ (2.1) are Killing's equations defining a motion.) Let $G_{r+1}$ be the full Lie group of homothetic mappings of $V_{n}$, the vectors of the group being $\xi_{(\alpha)}^{i}, \alpha=1,2, \cdots, r+1$, each vector defining a partial Lie derivative $L_{\alpha}$. Then $L_{\alpha} g_{i j}=c_{\alpha} g_{i j}$ and any vector $k^{\alpha} \xi_{\alpha}^{i}$ defines the mapping such that

$$
L g_{i j}=k^{\alpha} L_{\alpha} g_{i j}=k^{\alpha} c_{\alpha} g_{i j} .
$$

Now $c_{\alpha}$ are constants (not all $=0$ ) so that $k^{\alpha} c_{\alpha}=0$ admits $r$ independent solutions $k_{(\sigma)}^{\alpha}, \sigma=1, \cdots, r$. Hence without loss of generality we may choose as a basis the vectors

$$
\bar{\xi}_{(\sigma)}^{i}=k_{(\sigma)}^{\alpha} \xi_{(\alpha)}^{i}, \quad \eta^{i}=\xi_{(r+1)}^{i},
$$

so that $\bar{L}_{(\sigma)} g_{i j}=0, L_{r+1} g_{i j}=c g_{i j}$. This shows that $\bar{\xi}_{(\sigma)}^{i}$ define a $G_{r}$ group of motions of $V_{n}$. We prove that this $G_{r}$ is an invariant subgroup of $G_{r+1}$. To this end let $\xi^{i}$ be a vector of $G_{r}$; we must show that if $X$ $=\xi^{i} \partial / \partial x^{i}$ and $Y=\eta^{i} \partial / \partial x^{i}$ then $(X, Y)=c^{\sigma} X_{a}$. Let $(X, Y) f=Z f$ $=\zeta^{i} \partial f / \partial x^{i}$; then $\zeta^{i}=\xi^{h} \eta^{i}{ }_{, h}-\eta^{h} \xi^{i}{ }_{, h}$. We also know that $\xi^{i}$ and $\eta^{i}$ define affine collineations of $V_{n}$ so that $\xi_{, j, k}^{i}+\xi^{h} R_{j k h}^{i}=\eta_{, j, k}^{i}+\eta^{h} R_{j k h}^{i}=0$ so that

Received by the editors May 31, 1958. 


$$
\begin{aligned}
\zeta_{, j}^{i} & =\xi_{, j}^{h} \eta_{, h}^{i}-\eta_{, j}^{h} \xi_{, h}^{i}-\xi^{h} \eta^{l} R_{h j l}^{i}+\eta^{h} \xi^{l} R_{h j l}^{i} \\
& =\xi_{, j}^{h} \eta_{, h}^{i}-\eta_{, j}^{h} \xi_{, h}^{i}+\xi^{h} \eta^{l} R_{j l h}^{i}
\end{aligned}
$$

or

$$
\zeta_{k, j}=\xi_{, j}^{h} \eta_{k, h}-\eta_{, j}^{h} \xi_{k, h}+\xi^{h} \eta^{l} R_{k j l h} .
$$

Interchanging $k$ and $j$ and adding the two sets of equations and making use of (2.1), we find that

$$
\begin{aligned}
\zeta_{k, j}+\zeta_{j, k} & =g^{h l} \xi_{l, j}\left(\eta_{k, h}+\eta_{h, k}\right)+g^{h l} \xi_{l, k}\left(\eta_{j, h}+\eta_{h, j}\right) \\
& =c\left(\xi_{k, j}+\xi_{j, k}\right)=0,
\end{aligned}
$$

so that $\zeta^{i}$ defines a motion and is therefore in $G_{r}$. Thus we have

THEOREM 2.1. The full group $G_{r+1}$ of homothetic mappings of $V_{n}$ contains an invariant subgroup $G_{r}$ of motions and $a G_{1}$ subgroup of dilations.

Another theorem that may be proved in a similar manner is

Theorem 2.2. A Riemann space of constant nonzero curvature does not admit any homothetic mappings except motions.

This is a generalization of some theorems of Sumitomo, since it is independent of compactness or orientability.

\section{BiBLIOGRAPHY}

1. Takeshi Sumitomo, On some transformations of Riemann spaces, Tensor (New Series) vol. 6, no. 2 (1956).

2. M. S. Knebelman, On the equations of motion in Riemann spaces, Bull. Amer. Math. Soc. vol. 51, no. 10 (1945).

3. K. Yano and S. Bochner, Curvature and Betti Numbers, Annals of Mathematics Studies, no. 32, Princeton University Press, 1953.

Washington State College 